\title{
A note on Liouville theorem for stationary flows of shear thickening fluids in the plane *
}

\author{
Guo Zhang \\ Department of Mathematics and Statistics, P.O. Box 35 (MaD), FI-40014 \\ University of Jyväskylä, Finland
}

\begin{abstract}
In this paper we consider the entire weak solutions of the equations for stationary flows of shear thickening fluids in the plane and prove Liouville theorem under the global boundedness condition of velocity fields.
\end{abstract}

MR Subject Classification: 76 D 05, 76 D 07, 76 M 30, 35 Q 30.

Keywords: Shear thickening fluids, Entire weak solutions, Liouville theorem.

\section{$\S 1$. Introduction}

In this note, we study entire weak solutions $u: \mathbb{R}^{2} \rightarrow \mathbb{R}^{2}, \pi: \mathbb{R}^{2} \rightarrow \mathbb{R}$ of the following system

$$
\left\{\begin{array}{l}
-\operatorname{div}[T(\varepsilon(u))]+u^{k} \partial_{k} u+D \pi=0, \\
\operatorname{div} u=0 \quad \text { in } \mathbb{R}^{2}
\end{array}\right.
$$

and show that bounded solutions are constants.

The above system describes the stationary flow of an incompressible generalized Newtonian fluid, where $u$ is the velocity field, $\pi$ the pressure function, $u^{k} \partial_{k} u$ the convective term, and $T$ represents the stress deviator tensor. As usual $\varepsilon(u)$ stands for the symmetric part of the differential matrix $D u$ of $u$, i.e.

$$
\varepsilon(u)=\frac{1}{2}\left(D u+(D u)^{T}\right)=\frac{1}{2}\left(\partial_{i} u^{k}+\partial_{k} u^{i}\right)_{1 \leq i, k \leq 2} .
$$

we assume that the stress tensor $T$ is the gradient of a potential $H: S^{2 \times 2} \rightarrow \mathbb{R}$ defined on the space $S^{2 \times 2}$ of all symmetric $(2 \times 2)$ matrices of the following form

$$
H(\varepsilon)=h(|\varepsilon|),
$$

where $h$ is a nonnegative function of class $C^{2}$. Thus

$$
T(\varepsilon)=D H(|\varepsilon|)=\mu(|\varepsilon|) \varepsilon, \quad \mu(t)=\frac{h^{\prime}(t)}{t} .
$$

\footnotetext{
*E-mail addresses: guozhang@jyu.fi
} 
If $\mu$ is a constant, $\mu=\nu$, that is, if $h(t)=\frac{\nu}{2} t^{2}$, then system (1.1) reduces to the stationary Navier-Stokes equations for incompressible Newtonian fluids with viscosity coefficient $\nu$.

If $\mu$ is not a constant, then it means that the viscosity coefficient depends on $\varepsilon$, and system (1.1) describes the motion of continuous media of generalized Newtonian fluids. For the physical background and mathematical theory of generalized Newtonian fluids, we refer to Ladyzhenskaya Lad69, Galdi Gal94a, Gal94b, Malek, Necas, Rokyta and Ruzicka [MNRR96], and Fuchs and Seregin [FS00].

In the whole paper, we will concentrate on some special types of shear thickening fluids. We assume that the potential $h$ satisfies the following conditions:

$h$ is strictly increasing and convex

$$
\text { together with } h^{\prime \prime}(0)>0 \text { and } \lim _{t \rightarrow 0} \frac{h(t)}{t}=0 \text {; }
$$

(doubling property) there exists a constant $a \geq 1$

such that $h(2 t) \leq a h(t)$ for all $t \geq 0$;

$$
\text { we have } \frac{h^{\prime}(t)}{t} \leq h^{\prime \prime}(t) \text { for any } t \geq 0 \text {. }
$$

The study of Liouville type theorems goes back to the work of Gilbarg and Weinberger GW78. They showed that entire solutions $u$ of stationary Navier-Stokes equations in the plane are constants, provided that $\int_{\mathbb{R}^{2}}|D u|^{2} d x<\infty$. For the unstationary Navier-Stokes equations in 2D, recently, Koch, Nadirashvili, Seregin and Sverak [KNSS09] showed that $u(x, t)=b(t)$ on $\mathbb{R}^{2} \times(-\infty, 0)$ provided the solutions are bounded. Clearly, this result implies the Liouville theorem for stationary Navier-Stokes equations, that is, bounded solutions to stationary Navier-Stokes equations are constants.

In the case of shear thickening fluids, for $h$ satisfying $(A 1)-(A 3)$, Fuchs [Fuc] very recently proved the following Liouville theorem.

Theorem [Theorem 1.2, [Fuc]] Let $u \in C^{2}\left(\mathbb{R}^{2}, \mathbb{R}^{2}\right), \pi \in C^{1}\left(\mathbb{R}^{2}, \mathbb{R}\right)$ be the solutions to (1.1). Suppose that $u$ is bounded in $\mathbb{R}^{2}$ and satisfies that

$$
\sup _{\mathbb{R}^{2}-B_{R}(0)}\left|u-u_{\infty}\right| \rightarrow 0
$$

as $R \rightarrow \infty$, for a vector $u_{\infty} \in \mathbb{R}^{2}$. Then $u$ is a constant vector.

In Fuc, Fuchs conjectured that one can remove the assumption $(*)$ on $u$ at infinity in the above theorem and show that any bounded solution $u$ must be a constant vector. In this note, we will give a positive answer to this conjecture.

Theorem 1 Suppose $u \in C^{1}\left(\mathbb{R}^{2}, \mathbb{R}^{2}\right) \bigcap L^{\infty}\left(\mathbb{R}^{2}, \mathbb{R}^{2}\right)$ be an entire weak solution to (1.1), i.e.

$$
\int_{\mathbb{R}^{2}} T(\varepsilon(u)): \varepsilon(\varphi) d x-\int_{\mathbb{R}^{2}} u^{k} u^{i} \partial_{k} \varphi^{i} d x=0
$$

for all $\varphi \in C_{0}^{\infty}\left(\mathbb{R}^{2}, \mathbb{R}^{2}\right), \operatorname{div} \varphi=0$. Then $u$ is a constant vector.

We first comment on the regularity assumption on the solutions. As we known, for general $h$ satisfying $(A 1)-(A 3), C^{1, \alpha}$ regularity of solutions of (1.1) is an open problem, 
but in some special case, such as $h(t)=t^{2}(1+t)^{m}, m \geq 0$, the solution $u$ belongs to the space $C^{1, \alpha}$ [BFZ05], so the assumption in Theorem 1 is reasonable.

Second, we comment on the proof of Theorem 1. Our proof follows the same line as that of Theorem 1.2 in [Fuc]. We first need an energy estimate for the first order derivatives, as stated in Lemma 3.1. This was proved in [Fuc]. Then we need the energy estimate for the second order derivatives, see formula (3.10), from which follows that

$$
\int_{\mathbb{R}^{2}} D^{2} H(\varepsilon(u))\left(\varepsilon\left(\partial_{k} u\right), \varepsilon\left(\partial_{k} u\right)\right) d x<\infty .
$$

Actually, ( $\star$ ) was proved in [Fuc, via a Caccioppoli-type inequality, see (5.10) in [Fuc]. Our Caccioppoli-type inequality is different from (5.10) in [Fuc]. For the proof of (3.10), we use a different approach than that in $[\mathrm{Fuc}$ to estimate the item involving the pressure $\pi$. The treatment is standard. The essential point of our proof is based on the delicate analysis of Caccioppoli-type inequality (3.10). We estimate one crucial item involving the first order derivatives by integration by parts. This idea was also used in [FZ12].

Our notations is standard. Throughout this paper, the convention of summation with respect to indices repeated twice is used. All constants are denoted by the symbol $C$, and $C$ may change from line to line. Whenever it is necessary we will indicate the dependence of $C$ on parameters. As usual $Q_{R}\left(x_{0}\right)$ denotes the open square with center $x_{0}$ and side length $2 R$, and symbols :, · will be used for the scalar product of matrices and vectors respectively. $|\cdot|$ denotes the associated Euclidean norms.

Our paper is organized as follows: in Section 2 we present some auxiliary results, and in Section 3 we give the proof of Theorem 1.

\section{§2. Auxiliary Results}

As shown in Fuc, the following properties of functions $h$ follows from $(A 1)-(A 3)$.

(i) $\mu(t)=\frac{h^{\prime}(t)}{t}$ is an increasing function.

(ii) We have $h(0)=h^{\prime}(0)=0$ and

$$
h(t) \geq \frac{1}{2} h^{\prime \prime}(0) t^{2}
$$

Moreover,

$$
\frac{h^{\prime}(t)}{t} \geq \lim _{s \rightarrow 0} \frac{h^{\prime}(s)}{s}=h^{\prime \prime}(0)>0 .
$$

(iii) The function $h$ satisfies the balancing condition, i.e.

$$
\frac{1}{a} h^{\prime}(t) t \leq h(t) \leq t h^{\prime}(t), \quad t \geq 0 .
$$

(iv) For an exponent $m \geq 2$ and a constant $C \geq 0$ it holds

$$
h(t) \leq C\left(1+t^{m}\right), \quad h^{\prime}(t) \leq C\left(1+t^{m}\right), \quad t \geq 0 .
$$

From the assumptions on $h$, we know the system satisfies the following elliptic condition, $\forall \varepsilon, \sigma \in S^{2}$,

$$
\frac{h^{\prime}(|\varepsilon|)}{|\varepsilon|}|\sigma|^{2} \leq D^{2} H(\varepsilon)(\sigma, \sigma) \leq h^{\prime \prime}(|\varepsilon|)|\sigma|^{2},
$$


from which, together with (2.2), follows that

$$
D^{2} H(\varepsilon)(\sigma, \sigma) \geq h^{\prime \prime}(0)|\sigma|^{2} .
$$

In the proof of Theorem 1, we need the following results.

The first results is Lemma 3.1 in [FZ12, which is a slight extension of a result of Giaquinta and Modica GM82]. Define $Q_{R}\left(z_{0}\right)=\left\{(x, y) \in \mathbb{R}^{2},\left|x-x_{0}\right|<R,\left|y-y_{0}\right|<R\right\}$, where , $z_{0}=\left(x_{0}, y_{0}\right) \in \mathbb{R}^{2}$.

Lemma 2.1 Let $f, f_{1}, \ldots, f_{\ell}$ denote non-negative functions from the space $L_{l o c}^{1}\left(\mathbb{R}^{2}\right)$. Suppose further that we are given exponents $\alpha_{1}, \ldots, \alpha_{\ell}>0$. Then we can find a number $\delta_{0}>0$ depending on $\alpha_{1}, \ldots, \alpha_{\ell}$ as follows: if for $\delta \in\left(0, \delta_{0}\right)$ it is possible to calculate a constant $C(\delta)>0$ such that the inequality

$$
\int_{Q_{R}(z)} f d x \leq \delta \int_{Q_{2 R}(z)} f d x+C(\delta) \sum_{j=1}^{\ell} R^{-\alpha_{j}} \int_{Q_{2 R}(z)} f_{j} d x
$$

holds for any choice of $Q_{R}(z) \subset \mathbb{R}^{2}$, then there is a constant $C$ with the property

$$
\int_{Q_{R}(z)} f d x \leq C \sum_{j=1}^{\ell} R^{-\alpha_{j}} \int_{Q_{2 R}(z)} f_{j} d x
$$

for all squares $Q_{R}(z)$.

Next, we need a standard result concerning the "divergence equations", see e.g. Gal94a, Gal94b or Lad69.

Lemma 2.2 Consider a function $f \in L^{2}\left(Q_{R}(z)\right)$ such that $\int_{Q_{R}(z)} f d x=0$. Then there exists a field $v \in W_{0}^{1,2}\left(Q_{R}(z), \mathbb{R}^{2}\right)$ and a constant $C$ independent of $Q_{R}(z)$ such that we have $\operatorname{div} v=f$ on $Q_{R}(z)$ together with the estimate

$$
\int_{Q_{R}(z)}|D v|^{2} d x \leq C \int_{Q_{R}(z)} f^{2} d x .
$$

Finally, we need the $L^{2}$-variant of the classical Korn inequality.

Lemma 2.3 There is a constant $C$ independent of $Q_{R}(z)$ such that for all $v \in$ $W_{0}^{1,2}\left(Q_{R}(z), \mathbb{R}^{2}\right)$ it holds

$$
\int_{Q_{R}(z)}|D v|^{2} d x \leq C \int_{Q_{R}(z)}|\varepsilon(v)|^{2} d x .
$$

\section{§3. The Proof Of Theorem 1}

The following energy estimate was proved as Lemma 4.1 in [Fuc].

Proposition 3.1 Suppose that $u \in C^{2}\left(\mathbb{R}^{2}, \mathbb{R}^{2}\right)$ is a bounded solution to (1.1), where $h$ satisfies $(A 1)-(A 3)$. Then it holds

$$
\int_{B_{t}\left(x_{0}\right)} H(\varepsilon(u)) d x \leq C(t+1)
$$


for all discs $B_{t}\left(x_{0}\right) \subset \mathbb{R}^{2}$.

In the above Proposition 3.1, we can replace $u \in C^{2}\left(\mathbb{R}^{2}, \mathbb{R}^{2}\right)$ by $u \in C^{1}\left(\mathbb{R}^{2}, \mathbb{R}^{2}\right)$ and replace the discs $B_{t}\left(x_{0}\right)$ by the squares $Q_{t}\left(x_{0}\right)$. Indeed, if $u \in C^{1}\left(\mathbb{R}^{2}, \mathbb{R}^{2}\right)$, in view of the elliptic condition (2.5), the system is local uniformly elliptic, using the difference quote technique, it follows that $u \in W_{\text {loc }}^{2,2}\left(\mathbb{R}^{2}, \mathbb{R}^{2}\right) \cap C^{1}\left(\mathbb{R}^{2}, \mathbb{R}^{2}\right)$, see Fuc12. Furthermore, it follows that $\pi \in W_{\text {loc }}^{1,2}\left(\mathbb{R}^{2}, \mathbb{R}\right)$. Consequently, as mentioned in [Fuc], we can follow the proof of Lemma 4.1 in $[\mathrm{Fuc}$ to show that Lemma 3.1 holds under the assumption $u \in C^{1}\left(\mathbb{R}^{2}, \mathbb{R}^{2}\right)$.

Now we have $u \in W_{\text {loc }}^{2,2}\left(\mathbb{R}^{2}, \mathbb{R}^{2}\right) \bigcap C^{1}\left(\mathbb{R}^{2}, \mathbb{R}^{2}\right)$ and $\pi \in W_{\text {loc }}^{1,2}\left(\mathbb{R}^{2}, \mathbb{R}\right)$. Under the boundedness condition of the solution, we would like to prove the validity of

$$
\int_{\mathbb{R}^{2}} D^{2} H(\varepsilon(u))\left(\varepsilon\left(\partial_{k} u\right), \varepsilon\left(\partial_{k} u\right)\right) d x<\infty
$$

Note that from (2.6) and (3.1) it immediately follows that

$$
\int_{\mathbb{R}^{2}}|D \varepsilon(u)|^{2} d x<\infty
$$

To prove (3.1), we go back to the system (1.1). For any $\eta \in C_{0}^{\infty}\left(\mathbb{R}^{2}\right)$ with $0 \leq \eta \leq 1$, letting $\varphi_{k}=\partial_{k} u \eta^{2}$, we multiply (1.1) with $\partial_{k} \varphi_{k}$ and use integration by parts to obtain

$$
\int_{\mathbb{R}^{2}} \partial_{k} \sigma: \varepsilon\left(\varphi_{k}\right) d x-\int_{\mathbb{R}^{2}} D \pi \cdot \partial_{k} \varphi_{k} d x-\int_{\mathbb{R}^{2}} u^{i} \partial_{i} u \cdot \partial_{k} \varphi_{k} d x=0
$$

where $\sigma:=D H(\varepsilon(u)):=\frac{h^{\prime}(|\varepsilon(u)|)}{|\varepsilon(u)|} \varepsilon(u)$.

Recalling $\varphi=\partial_{k} u \eta^{2}$ and using integration by parts again, we obtain

$$
\begin{aligned}
\int_{\mathbb{R}^{2}} \partial_{k} \sigma: \varepsilon\left(\partial_{k} u\right) \eta^{2} d x & =\int_{\mathbb{R}^{2}} \sigma: \partial_{k}\left(D \eta^{2} \odot \partial_{k} u\right) d x+\int_{\mathbb{R}^{2}} \partial_{k} \pi \operatorname{div}\left(\varphi_{k}\right) d x \\
& +\int_{\mathbb{R}^{2}} u^{i} \partial_{i} u \cdot \partial_{k} \varphi_{k} d x=: I+I I+I I I
\end{aligned}
$$

where $\odot$ is the symmetric product of vectors.

In the following, we will deal with all of the terms in (3.3). For the left hand side, we have

$$
\int_{\mathbb{R}^{2}} \partial_{k} \sigma: \varepsilon\left(\partial_{k} u\right) \eta^{2} d x=\int_{\mathbb{R}^{2}} D^{2} H(\varepsilon(u))\left(\varepsilon\left(\partial_{k} u\right), \varepsilon\left(\partial_{k} u\right)\right) \eta^{2} d x
$$

We will estimate the items $I, I I I$ in the same way as that in [Fuc]. For the completeness, we include the proofs here.

For I, using Young's inequality and the estimates (2.3) and (2.5) we have for any 
$\delta>0$,

$$
\begin{aligned}
& I=\int_{\mathbb{R}^{2}} \sigma: \partial_{k}\left(D\left(\eta^{2}\right) \odot \partial_{k} u\right) d x \\
& \leq C\left\{\int_{\mathbb{R}^{2}} h^{\prime}(|\varepsilon(u)|)|D u|\left(|D \eta|^{2}+\left|D^{2} \eta\right|\right) d x+\int_{\mathbb{R}^{2}} h^{\prime}(|\varepsilon(u)|)|D \eta| \eta\left|D^{2} u\right| d x\right\} \\
& \leq \delta \int_{\mathbb{R}^{2}} \frac{h^{\prime}(|\varepsilon(u)|)}{|\varepsilon(u)|}|D \varepsilon(u)|^{2} \eta^{2} d x+C(\delta) \int_{\mathbb{R}^{2}} h^{\prime}(|\varepsilon(u)|)|\varepsilon(u)||D \eta|^{2} d x \\
& +C \int_{\mathbb{R}^{2}}|D u|^{2}\left(|D \eta|^{2}+\left|D^{2} \eta\right|\right) d x+C \int_{\mathbb{R}^{2}} h^{\prime}(|\varepsilon(u)|)^{2}\left(|D \eta|^{2}+\left|D^{2} \eta\right|\right) \\
& \leq \delta \int_{\mathbb{R}^{2}} D^{2} H(\varepsilon(u))\left(\partial_{k} \varepsilon(u), \partial_{k} \varepsilon(u)\right) \eta^{2} d x+C(\delta) \int_{\mathbb{R}^{2}} h(|\varepsilon(u)|)|D \eta|^{2} d x \\
& +C \int_{\mathbb{R}^{2}} h^{\prime}(|\varepsilon(u)|)^{2}\left(|D \eta|^{2}+\left|D^{2} \eta\right|\right) d x+C \int_{\mathbb{R}^{2}}|D u|^{2}\left(|D \eta|^{2}+\left|D^{2} \eta\right|\right) d x,
\end{aligned}
$$

where the relation $\left|D^{2} u(x)\right| \leq C|D \varepsilon(u)(x)|$ is used in the second inequality.

For $I I I$ we have identity

$$
\begin{aligned}
I I I & =\int_{\mathbb{R}^{2}} u^{i} \partial_{i} u^{j} \partial_{k}\left(\partial_{k} u^{j} \eta^{2}\right) d x=-\int_{\mathbb{R}^{2}} \partial_{k}\left(u^{i} \partial_{i} u^{j}\right) \partial_{k} u^{j} \eta^{2} d x \\
& =-\int_{\mathbb{R}^{2}} \partial_{k} u^{i} \partial_{i} u^{j} \partial_{k} u^{j} \eta^{2} d x-\int_{\mathbb{R}^{2}} u^{i} \frac{1}{2} \partial_{i}\left(\left|\partial_{k} u^{j}\right|^{2}\right) \eta^{2} d x \\
& =\frac{1}{2} \int_{\mathbb{R}^{2}}|D u|^{2} u \cdot D \eta^{2} d x
\end{aligned}
$$

where we use the identity $\partial_{k} u^{i} \partial_{i} u^{j} \partial_{k} u^{j}=0$ for divergence free vector $u$ in $2 D$.

Finally, we estimate $I I$. Here we just use equation (1.1) to replace $D \pi$.

$$
\begin{aligned}
I I & =\int_{\mathbb{R}^{2}} \partial_{k} \pi \operatorname{div}\left(\varphi_{k}\right) d x=\int_{\mathbb{R}^{2}} \partial_{k} \pi \partial_{k} u \cdot D \eta^{2} d x \\
& =-\int_{\mathbb{R}^{2}} \sigma_{i k} \partial_{i}\left(\partial_{k} u \cdot D \eta^{2}\right) d x-\int_{\mathbb{R}^{2}} u^{i} \partial_{i} u^{k} \partial_{k} u \cdot D \eta^{2} d x .
\end{aligned}
$$

We estimate the first integral of (3.7) in the same way as that in the above for $I$ we have

$$
\begin{aligned}
I I & \leq \delta \int_{\mathbb{R}^{2}} D^{2} H(\varepsilon(u))\left(\varepsilon\left(\partial_{k} u\right), \varepsilon\left(\partial_{k} u\right)\right) \eta^{2} d x \\
& +C(\delta) \int_{\mathbb{R}^{2}} h(|\varepsilon(u)|)|D \eta|^{2} d x+\int_{\mathbb{R}^{2}} h^{\prime}(|\varepsilon(u)|)^{2}\left(|D \eta|^{2}+\left|D^{2} \eta\right|\right) d x \\
& +\int_{\mathbb{R}^{2}}|D u|^{2}\left(|D \eta|^{2}+\left|D^{2} \eta\right|\right) d x+C \int_{\mathbb{R}^{2}}|D u|^{2}|u||D \eta| d x .
\end{aligned}
$$

Combining (3.3), (3.4), (3.5), (3.6) and (3.8) and choosing $\delta=\frac{1}{4}$, we end up with

$$
\begin{aligned}
& \int_{\mathbb{R}^{2}} D^{2} H(\varepsilon(u))\left(\varepsilon\left(\partial_{k} u\right), \varepsilon\left(\partial_{k} u\right)\right) \eta^{2} d x \\
& \leq C\left\{\int_{\mathbb{R}^{2}} h(|\varepsilon(u)|)|D \eta|^{2} d x+\int_{\mathbb{R}^{2}} h^{\prime}(|\varepsilon(u)|)^{2}\left(|D \eta|^{2}+\left|D^{2} \eta\right|\right) d x\right. \\
& \left.+\int_{\mathbb{R}^{2}}|D u|^{2}\left(|D \eta|^{2}+\left|D^{2} \eta\right|\right) d x+\int_{\mathbb{R}^{2}}|D u|^{2}|u||D \eta| d x\right\} .
\end{aligned}
$$


Now, choosing $\eta \in C_{0}^{\infty}\left(Q_{\frac{3}{2} R}\left(x_{0}\right)\right)$ such that $\eta \equiv 1$ in $Q_{R}\left(x_{0}\right),|D \eta| \leq \frac{4}{R}$, and $\left|D^{2} \eta\right| \leq$ $\frac{16}{R^{2}}$ we obtain from (3.9) that

$$
\begin{aligned}
\int_{Q_{R}\left(x_{0}\right)} w d x & \leq C\left\{\frac{1}{R^{2}} \int_{Q_{\frac{3}{2} R}\left(x_{0}\right)} h(|\varepsilon(u)|) d x+\frac{1}{R^{2}} \int_{Q_{\frac{3}{2} R}\left(x_{0}\right)} h^{\prime}(|\varepsilon(u)|)^{2} d x\right. \\
& \left.+\frac{1}{R^{2}} \int_{Q_{\frac{3}{2} R}\left(x_{0}\right)}|D u|^{2} d x\right\}+\frac{C\left(\|u\|_{L_{\infty}}\right)}{R} \int_{T_{\frac{3}{2} R}\left(x_{0}\right)}|D u|^{2} d x
\end{aligned}
$$

where $w:=D^{2} H(\varepsilon(u))\left(\varepsilon\left(\partial_{k} u\right), \varepsilon\left(\partial_{k} u\right)\right)$ and $T_{\frac{3}{2} R}\left(x_{0}\right)=Q_{\frac{3}{2} R}\left(x_{0}\right) \backslash \overline{Q_{R}\left(x_{0}\right)}$.

We will show that it follows from (3.10) that (3.1) holds. The proof is the same as in [Fuc]. For the completeness, we conclude the proof here, some steps are a little bit different from those in Fuc.

Let $\xi \in C_{0}^{\infty}\left(Q_{2 R}\left(x_{0}\right)\right)$ be the cut-off function such that, $0 \leq \xi \leq 1, \xi \equiv 1$ on $Q_{\frac{3}{2} R}\left(x_{0}\right)$ and $|D \xi| \leq \frac{4}{R}$. We have by Lemma 2.3 that

$$
\begin{aligned}
\int_{Q_{\frac{3}{2} R}\left(x_{0}\right)}|D u|^{2} d x & \leq \int_{Q_{2 R}\left(x_{0}\right)} \xi^{2}|D u|^{2} d x \\
& \leq C\left(\int_{Q_{2 R}\left(x_{0}\right)}|D(u \xi)|^{2} d x+\int_{Q_{2 R}\left(x_{0}\right)}|u|^{2}|D \xi|^{2} d x\right) \\
& \leq C\left(\int_{Q_{2 R}\left(x_{0}\right)}|\varepsilon(u \xi)|^{2} d x+\int_{Q_{2 R}\left(x_{0}\right)}|u|^{2}|D \xi|^{2} d x\right) \\
& \leq C\left(\int_{Q_{2 R}\left(x_{0}\right)}|\varepsilon(u)|^{2} d x+\frac{1}{R^{2}} \int_{Q_{2 R}\left(x_{0}\right)}|u|^{2} d x\right)
\end{aligned}
$$
that

Now, since $\frac{h^{\prime}(t)}{t}$ is an increasing function and $h$ satisfies (2.3), for any $L>0$, it follows

$$
\begin{aligned}
& \int_{Q_{\frac{3}{2} R}\left(x_{0}\right)} h^{\prime}(|\varepsilon(u)|)^{2} d x \\
& \leq \int_{\left\{x \in Q_{\frac{3}{2} R}\left(x_{0}\right),|\varepsilon(u)| \leq L\right\}} h^{\prime}(L)^{2} d x+\int_{\left\{x \in Q_{\frac{3}{2} R}\left(x_{0}\right),|\varepsilon(u)|>L\right\}} h^{\prime}(|\varepsilon(u)|)^{2} d x \\
& \leq C R^{2} h^{\prime}(L)^{2}+\frac{C}{L^{2}} \int_{Q_{\frac{3}{2} R}\left(x_{0}\right)} h(|\varepsilon(u)|)^{2} d x .
\end{aligned}
$$

By Sobolev inequality we have

$$
\begin{aligned}
& \int_{Q_{\frac{3}{2} R}\left(x_{0}\right)} h(|\varepsilon(u)|)^{2} d x \leq C\left(\int_{Q_{2 R}\left(x_{0}\right)}|D(\xi h(|\varepsilon(u)|))| d x\right)^{2} \\
& \leq C\left(\int_{Q_{2 R}\left(x_{0}\right)}|D \xi| h(|\varepsilon(u)|) d x\right)^{2}+C\left(\int_{Q_{2 R}\left(x_{0}\right)} \xi h^{\prime}(|\varepsilon(u)|)|D \varepsilon(u)| d x\right)^{2} .
\end{aligned}
$$


To estimate the second term in the above inequality, by Hölder inequality we have

$$
\begin{aligned}
& \left(\int_{Q_{2 R}\left(x_{0}\right)} \xi h^{\prime}(|\varepsilon(u)|)|D \varepsilon(u)| d x\right)^{2} \\
& \leq \int_{Q_{2 R}\left(x_{0}\right)} \xi h^{\prime}(|\varepsilon(u)|)|\varepsilon(u)| d x \int_{Q_{2 R}\left(x_{0}\right)} \xi \frac{h^{\prime}(|\varepsilon(u)|)}{|\varepsilon(u)|}|D \varepsilon(u)|^{2} d x .
\end{aligned}
$$

Combining the estimates (3.13) and (3.14) and recalling the definitions of $\xi$ and $w$ we have

$$
\begin{aligned}
& \int_{Q_{\frac{3}{2} R}\left(x_{0}\right)} h(|\varepsilon(u)|)^{2} d x \\
& \leq C \frac{1}{R^{2}}\left(\int_{Q_{2 R}\left(x_{0}\right)} h(|\varepsilon(u)|) d x\right)^{2}+C \int_{Q_{2 R}\left(x_{0}\right)} h(|\varepsilon(u)|) d x \int_{Q_{2 R}\left(x_{0}\right)} w d x .
\end{aligned}
$$

Thus (3.12) and (3.15) give us

$$
\begin{aligned}
\int_{Q_{\frac{3}{2} R}\left(x_{0}\right)} h^{\prime}(|\varepsilon(u)|)^{2} d x & \leq C R^{2} h^{\prime}(L)^{2}+C \frac{1}{L^{2}} \frac{1}{R^{2}}\left(\int_{Q_{2 R}\left(x_{0}\right)} h(|\varepsilon(u)|) d x\right)^{2} \\
& +C \frac{1}{L^{2}} \int_{Q_{2 R}\left(x_{0}\right)} h(|\varepsilon(u)|) d x \int_{Q_{2 R}\left(x_{0}\right)} w d x
\end{aligned}
$$

Then it follows from (3.10), (3.11) and (3.16) that

$$
\begin{aligned}
\int_{Q_{R}\left(x_{0}\right)} w d x & \leq C h^{\prime}(L)^{2}+C \frac{1}{L^{2}} \frac{1}{R^{2}} \int_{Q_{2 R}\left(x_{0}\right)} h(|\varepsilon(u)|) d x \int_{Q_{2 R}\left(x_{0}\right)} w d x \\
& +C \frac{1}{L^{2}} \frac{1}{R^{4}}\left(\int_{Q_{2 R}\left(x_{0}\right)} h(|\varepsilon(u)|) d x\right)^{2}+C \frac{1}{R^{2}} \int_{Q_{2 R}\left(x_{0}\right)} h(|\varepsilon(u)|) d x \\
& +C \frac{1}{R} \int_{Q_{2 R}\left(x_{0}\right)} h(|\varepsilon(u)|) d x+C \frac{1}{R^{4}} \int_{Q_{2 R}\left(x_{0}\right)}|u|^{2} d x \\
& +C \frac{1}{R^{3}} \int_{Q_{2 R}\left(x_{0}\right)}^{|u|^{2} d x}
\end{aligned}
$$

Now we choose $L=\frac{1}{R \beta}, \beta>0$. Then (3.17) gives

$$
\begin{aligned}
\int_{Q_{R}\left(x_{0}\right)} w d x & \leq C h^{\prime}\left(\frac{1}{R \beta}\right)^{2}+C \beta^{2} \int_{Q_{2 R}\left(x_{0}\right)} h(|\varepsilon(u)|) d x \int_{Q_{2 R}\left(x_{0}\right)} w d x \\
& +C \frac{\beta^{2}}{R^{2}}\left(\int_{Q_{2 R}\left(x_{0}\right)} h(|\varepsilon(u)|) d x\right)^{2}+C \frac{1}{R^{2}} \int_{Q_{2 R}\left(x_{0}\right)} h(|\varepsilon(u)|) d x \\
& +C \frac{1}{R} \int_{Q_{2 R}\left(x_{0}\right)} h(|\varepsilon(u)|) d x+C \frac{1}{R^{4}} \int_{Q_{2 R}\left(x_{0}\right)}|u|^{2} d x \\
& +C \frac{1}{R^{3}} \int_{Q_{2 R}\left(x_{0}\right)}^{|u|^{2} d x .}
\end{aligned}
$$


Letting $\beta^{2}=\frac{\varepsilon}{R+1}, \varepsilon<1$ in (3.18) and applying Proposition 3.1 we obtain that

$$
\begin{aligned}
& \int_{Q_{R}\left(x_{0}\right)} w d x \leq C \varepsilon \int_{Q_{2 R}\left(x_{0}\right)} w d x+C h^{\prime}\left(\frac{(R+1)^{\frac{1}{2}}}{\varepsilon^{\frac{1}{2}} R}\right)^{2} \\
& +C \frac{1}{R^{2}} \int_{Q_{2 R}\left(x_{0}\right)} h(|\varepsilon(u)|) d x+C \frac{1}{R} \int_{Q_{2 R}\left(x_{0}\right)} h(|\varepsilon(u)|) d x \\
& +C \frac{1}{R^{4}} \int_{Q_{2 R}\left(x_{0}\right)}|u|^{2} d x+C \frac{1}{R^{3}} \int_{Q_{2 R}\left(x_{0}\right)}|u|^{2} d x .
\end{aligned}
$$
have

Taking into account of (2.4) and choosing $\varepsilon$ small enough such that $\delta:=C \varepsilon<\frac{1}{2}$ we

$$
\begin{aligned}
& \int_{Q_{R}\left(x_{0}\right)} w d x \leq \delta \int_{Q_{2 R}\left(x_{0}\right)} w d x+C\left(1+\frac{1}{R^{2 m}}\right) \\
& +C \frac{1}{R^{2}} \int_{Q_{2 R}\left(x_{0}\right)} h(|\varepsilon(u)|) d x+C \frac{1}{R} \int_{Q_{2 R}\left(x_{0}\right)} h(|\varepsilon(u)|) d x \\
& +C \frac{1}{R^{4}} \int_{Q_{2 R}\left(x_{0}\right)}|u|^{2} d x+C \frac{1}{R^{3}} \int_{Q_{2 R}\left(x_{0}\right)}|u|^{2} d x,
\end{aligned}
$$

which, by Lemma 2.1, give us

$$
\begin{aligned}
\int_{Q_{R}\left(x_{0}\right)} w d x & \leq C\left(1+\frac{1}{R^{2 m}}\right)+C \frac{1}{R^{2}} \int_{Q_{2 R}\left(x_{0}\right)} h(|\varepsilon(u)|) d x \\
& +C \frac{1}{R} \int_{Q_{2 R}\left(x_{0}\right)} h(|\varepsilon(u)|) d x+C \frac{1}{R^{4}} \int_{Q_{2 R}\left(x_{0}\right)}|u|^{2} d x \\
& +C \frac{1}{R^{3}} \int_{Q_{2 R}\left(x_{0}\right)}^{|u|^{2} d x .}
\end{aligned}
$$

We apply Proposition 3.1 again and let $R \rightarrow \infty$ in (3.21). we conclude the proof of (3.1).

In the rest of the proof we show that

$$
\int_{\mathbb{R}^{2}} w d x=0
$$

Then it follows from (3.22) that $D \varepsilon(u)=0$. And hence $D^{2} u=0$. so $u$ is affine. Because we assume $u \in L^{\infty}\left(\mathbb{R}^{2}, \mathbb{R}^{2}\right)$, the claim of Theorem 1 follows.

Now it remains to prove (3.22). Let

$$
w_{\infty}:=\int_{\mathbb{R}^{2}} w d x
$$

Let us return to inequality (3.10). To prove (3.22), we need a delicate estimate for the integral $\int_{T_{\frac{3}{2} R}\left(x_{0}\right)}|D u|^{2} d x$. We choose a cut-off function $\zeta \in C_{0}^{\infty}\left(Q_{2 R}\left(x_{0}\right)\right), 0 \leq \zeta \leq 1, \zeta \equiv 1$ on $T_{\frac{3}{2} R}\left(x_{0}\right)$, spt $\zeta \subset T_{2 R}\left(x_{0}\right):=Q_{2 R}\left(x_{0}\right) \backslash \overline{Q_{\frac{R}{2}}\left(x_{0}\right)}$. By Lemma 2.3, we have

$$
\begin{aligned}
& \int_{T_{\frac{3}{2} R}\left(x_{0}\right)}|D u|^{2} d x \leq \int_{Q_{2 R}\left(x_{0}\right)}|D(u \zeta)|^{2} d x \leq C \int_{Q_{2 R}\left(x_{0}\right)}|\varepsilon(u \zeta)|^{2} d x \\
& \leq C \int_{Q_{2 R}\left(x_{0}\right)}|u|^{2}|D \zeta|^{2} d x+C \int_{Q_{2 R}\left(x_{0}\right)} \varepsilon_{i j}(u) \varepsilon_{i j}(u) \zeta^{2} d x .
\end{aligned}
$$


For the item $\int_{Q_{2 R}\left(x_{0}\right)} \varepsilon_{i j}(u) \varepsilon_{i j}(u) \zeta^{2} d x$, we use the energy for the second order derivative to control it. By integration by parts and Hölder inequality we have

$$
\begin{aligned}
& \int_{Q_{2 R}\left(x_{0}\right)} \varepsilon_{i j}(u) \varepsilon_{i j}(u) \zeta^{2} d x \\
& =-\int_{Q_{2 R}\left(x_{0}\right)} u^{i} \partial_{j}\left(\varepsilon_{i j}(u)\right) \zeta^{2} d x-\int_{Q_{2 R}\left(x_{0}\right)} u^{i} \varepsilon_{i j}(u) \partial_{j} \zeta^{2} d x \\
& \leq\left(\int_{Q_{2 R}\left(x_{0}\right)}|u|^{2} d x\right)^{\frac{1}{2}}\left(\int_{Q_{2 R}\left(x_{0}\right)}|D \varepsilon(u)|^{2} \zeta^{4} d x\right)^{\frac{1}{2}} \\
& +\left(\int_{Q_{2 R}\left(x_{0}\right)}|u|^{2}\left|D \zeta^{2}\right|^{2} d x\right)^{\frac{1}{2}}\left(\int_{Q_{2 R}\left(x_{0}\right)}|\varepsilon(u)|^{2} d x\right)^{\frac{1}{2}}
\end{aligned}
$$

Putting together the estimates (3.23) and (3.24) and recalling the definition of $\zeta$ we obtain

$$
\begin{aligned}
\int_{T_{\frac{3}{2} R}\left(x_{0}\right)}|D u|^{2} d x & \leq C \frac{1}{R^{2}} \int_{Q_{2 R}\left(x_{0}\right)}|u|^{2} d x \\
& +C \frac{1}{R}\left(\int_{Q_{2 R}\left(x_{0}\right)}|u|^{2} d x\right)^{\frac{1}{2}}\left(\int_{Q_{2 R}\left(x_{0}\right)}|\varepsilon(u)|^{2} d x\right)^{\frac{1}{2}} \\
& +C\left(\int_{Q_{2 R}\left(x_{0}\right)}|u|^{2} d x\right)^{\frac{1}{2}}\left(\int_{T_{2 R}\left(x_{0}\right)}|D \varepsilon(u)|^{2} d x\right)^{\frac{1}{2}} .
\end{aligned}
$$

Then it follows from (3.10), (3.11), (3.16) and (3.25) that

$$
\begin{aligned}
\int_{Q_{R}\left(x_{0}\right)} w d x & \leq C h^{\prime}(L)^{2}+C \frac{1}{L^{2}} \frac{1}{R^{2}} \int_{Q_{2 R}\left(x_{0}\right)} h(|\varepsilon(u)|) d x \int_{Q_{2 R}\left(x_{0}\right)} w d x \\
& +C \frac{1}{L^{2}} \frac{1}{R^{4}}\left(\int_{Q_{2 R}\left(x_{0}\right)} h(|\varepsilon(u)|) d x\right)^{2}+C \frac{1}{R^{2}} \int_{Q_{2 R}\left(x_{0}\right)} h(|\varepsilon(u)|) d x \\
& +C \frac{1}{R^{2}}\left(\int_{Q_{2 R}\left(x_{0}\right)}|u|^{2} d x\right)^{\frac{1}{2}}\left(\int_{Q_{2 R}\left(x_{0}\right)} h(|\varepsilon(u)|) d x\right)^{\frac{1}{2}} \\
& +C \frac{1}{R}\left(\int_{Q_{2 R}\left(x_{0}\right)}|u|^{2} d x\right)^{\frac{1}{2}}\left(\int_{T_{2 R}\left(x_{0}\right)}|D \varepsilon(u)|^{2} d x\right)^{\frac{1}{2}} \\
& +C \frac{1}{R^{3}} \int_{Q_{2 R}\left(x_{0}\right)}|u|^{2} d x+C \frac{1}{R^{4}} \int_{Q_{2 R}\left(x_{0}\right)}|u|^{2} d x,
\end{aligned}
$$

from which, repeating the above steps (3.18), (3.19) and (3.20) it follows that 


$$
\begin{aligned}
& \int_{Q_{R}\left(x_{0}\right)} w d x \leq \frac{1}{2} \int_{Q_{2 R}\left(x_{0}\right)} w d x+C h^{\prime}\left(\frac{(R+1)^{\frac{1}{2}}}{\varepsilon^{\frac{1}{2}} R}\right)^{2}+C \frac{1}{R^{2}} \int_{Q_{2 R}\left(x_{0}\right)} h(|\varepsilon(u)|) d x \\
& +C \frac{1}{R^{2}}\left(\int_{Q_{2 R}\left(x_{0}\right)}|u|^{2} d x\right)^{\frac{1}{2}}\left(\int_{Q_{2 R}\left(x_{0}\right)} h(|\varepsilon(u)|) d x\right)^{\frac{1}{2}} \\
& +C \frac{1}{R}\left(\int_{Q_{2 R}\left(x_{0}\right)}|u|^{2} d x\right)^{\frac{1}{2}}\left(\int_{T_{2 R}\left(x_{0}\right)}|D \varepsilon(u)|^{2} d x\right)^{\frac{1}{2}} \\
& +C \frac{1}{R^{3}} \int_{Q_{2 R}\left(x_{0}\right)}|u|^{2} d x+C \frac{1}{R^{4}} \int_{Q_{2 R}\left(x_{0}\right)}|u|^{2} d x
\end{aligned}
$$

now let $R$ go to infinity. Then from which, together with the boundedness condition of the solution $u, h^{\prime}(0)=0,(3.2)$ and Proposition 3.1, gives us

$$
w_{\infty} \leq \frac{1}{2} w_{\infty}
$$

Thus $w_{\infty}=0$. The proof is complete.

Acknowledgement The author wants to thank Martin Fuchs for many discussions. This author was supported by the Academy of Finland.

\section{References}

[BFZ05] M. Bildhauer, M. Fuchs, and X. Zhong. A lemma on the higher integrability of functions with applications to the regularity theory of two-dimensional generalized Newtonian fluids. Manuscripta Math., 116(2):135-156, 2005.

[FS00] M. Fuchs and G. Seregin. Variational methods for problems from plasticity theory and for generalized Newtonian fluids, volume 1749 of Lecture Notes in Mathematics. Springer-Verlag, Berlin, 2000.

[Fuc] M. Fuchs. Liouville theorems for stationary flows of shear thickening fluids in the plane. J. Math. Fluid Mech. To appear.

[Fuc12] M. Fuchs. Stationary flows of shear thickening fluids in 2D. J. Math. Fluid Mech., 14(1):43-54, 2012.

[FZ12] M. Fuchs and G. Zhang. Liouville theorems for entire local minimizers of energies defined on the class $L \log L$ and for entire solutions of the stationary Prandtl-Eyring fluid model. Calc. Var. Partial Differential Equations, 44(12):271-295, 2012.

[Gal94a] G. P. Galdi. An introduction to the mathematical theory of the NavierStokes equations. Vol. I, volume 38 of Springer Tracts in Natural Philosophy. Springer-Verlag, New York, 1994. Linearized steady problems. 
[Gal94b] G. P. Galdi. An introduction to the mathematical theory of the NavierStokes equations. Vol. II, volume 39 of Springer Tracts in Natural Philosophy. Springer-Verlag, New York, 1994. Nonlinear steady problems.

[GM82] M. Giaquinta and G. Modica. Nonlinear systems of the type of the stationary Navier-Stokes system. J. Reine Angew. Math., 330:173-214, 1982.

[GW78] D. Gilbarg and H. F. Weinberger. Asymptotic properties of steady plane solutions of the Navier-Stokes equations with bounded Dirichlet integral. Ann. Scuola Norm. Sup. Pisa Cl. Sci. (4), 5(2):381-404, 1978.

[KNSS09] G. Koch, N. Nadirashvili, G. A. Seregin, and V. Sverak. Liouville theorems for the Navier-Stokes equations and applications. Acta Math., 203(1):83-105, 2009.

[Lad69] O. A. Ladyzhenskaya. The mathematical theory of viscous incompressible flow. Second English edition, revised and enlarged. Translated from the Russian by Richard A. Silverman and John Chu. Mathematics and its Applications, Vol. 2. Gordon and Breach Science Publishers, New York, 1969.

[MNRR96] J. Malek, J. Necas, M. Rokyta, and M. Ruzicka. Weak and measure-valued solutions to evolutionary PDEs, volume 13 of Applied Mathematics and Mathematical Computation. Chapman \& Hall, London, 1996. 